Im Betriebe fließt die Flüssigkeit in der Regel ohne besondere Drosselung aus dem Druckrohr aus, aber in einer gewissen Höhe $h$ über dem Saugbehälterspiegel. Hierbei tritt die Förderhöhe h an Stelle ron $h_{0}$ in die Rechnung ein. Somit wird:

$$
\begin{gathered}
\mathrm{Q}=0,37 \cdot 0,20 \cdot(i 01 \cdot 1072 \cdot 10(\mathrm{H}-\mathrm{h}) \\
=2001 \mathrm{H}-\mathrm{h}
\end{gathered}
$$

die wirkliche Fördermenge der Pumpe ausdrücken.

Der Kraftbedarf dürfte etwa 1 PS. ausmachen. Wemn die Leergangsarbeit (bei abgeschlossener Leitung) $N_{0}$ schätzungsweise zu 0.5 PS. angenommen wird, läßt sich der Kraftbedarf aus $\mathrm{N}_{\mathrm{a}}=\mathrm{N}_{\mathrm{o}}+\mathrm{QH} / 60.75$ berechnen. Der Wirkungsorad $\varepsilon$ oder das Verhältnis der Nutzleistung Qh/60.75 zu dem Kraftbedarf $\mathrm{N}_{\mathrm{a}}$ wird am günstigsten, wenn $h=0.7 \mathrm{H}$ bis $0,9 \mathrm{H}$ ist.

In Fig. 3 ist der Verlauf der Werte von h für $\mathrm{H}=10 \mathrm{mWs}$. aufgezeichnet, ferner die zugehörigen Werte ron $Q$ und ron Qh (mit einem Maximum bei $h=\frac{2}{3} \mathrm{H}$ ), srhtieBlich auch $N_{\mathrm{a}}$ und $\varepsilon$. Die Größen sind für die Grundmaße a $/ b$ mit $u=0,74=2 v^{\prime}$ berechnet. Von praktischer Bedeutung sind nur die ersten Yaße bis höchstens $a / b=\frac{1}{3}$.

Fig. 4 zeigt für die Pumpe, in gleicher Weise wie Fig. 2 für den Fxhaustor, den Zusammenhang der̈ wichtiơsten Größen : Z. B. sei $Q=200 \mathrm{l} / \mathrm{L}$. und $\mathrm{h}=9 \mathrm{~m}$ Fördeshöhe für die verlangte Leistung gegeben. Nan findet rechts nach unten aufgetragen den Wert $Q=200$ bei der Strömung $c$ $=1,65 \mathrm{~m} \mathrm{~s}$ : dariiber bis zu der flach verlaufenden Parabel $\mathrm{H}-\mathrm{h}=1 \mathrm{~m}$. Geht man um $h=9 \mathrm{~m} n \mathrm{n} \cdot \mathrm{h}$ oben hinauf. so erreicht man die ganze Höhe $\mathrm{H}=10 \mathrm{~m}$ und geht in dieser Höhe nach links hinuiber. bis die link aufsteigende Parabel geschnitten wird; das geschieht bei dem nach links aufgetragenen Grunimals $n=950 \quad \mathrm{C} / \mathrm{Min}$., wobei die Radumfangsgeschwindigkeit $u=15 \mathrm{~m} / \mathrm{s}$ wird.

Die Zahlenwerte sind, nach den oben entwickelten Formeln. in folgender Tabelle auf-

\begin{tabular}{|c|c|c|c|c|c|c|c|c|c|}
\hline & & $\mathrm{h}=0$ & 2 & 4 & 6 & 8 & 10 & $1211 \mathrm{~W}$ & \\
\hline $\mathrm{Q}=0 \mathrm{M} \mathrm{M}$ & & $\mathrm{n}=0$ & 425 & 600 & 735 & 850 & 950 & $1040 \mathrm{~L} \mathrm{II}$ & $\mathrm{H}-\mathrm{h}=0 \mathrm{~m}$ \\
\hline 100 & $\mathrm{c}=0,8.3 \mathrm{~ms}$ & 150 & 450 & 620 & 750 & 860 & 960 & 1050 & 0,25 \\
\hline 150 & 1,25 & 225 & 480 & 640 & 760 & 8.0 & 975 & $10 b 0$ & 0,56 \\
\hline 200 & 1,67 & 300 & 520 & 670 & 790 & 900 & 995 & 1080 & 1,00 \\
\hline 250 & 2,08 & 375 & 565 & 705 & 810 & 925 & $10=0$ & 1100 & 1,56 \\
\hline 300 & 2,50 & 450 & 620 & 725 & 860 & 960 & 1050 & 1130 & 2,25 \\
\hline
\end{tabular}
gefuihrt :
Zum Schlul3 dieses Berichtes möchte ich nicht unterlassen, der Deutschen Steinzeugwarenfabrik in Friedrichsfeld i. B. die Anerkennuug dafür auszusprechen, daß sie sich entschlossen hat, sowoh] im allgemeinen als auch im besonderen Interesse der Benutzer ihrer Maschinen, die Ergebnisse aus den Untersuchungen ihrer Maschinen rückhaltlos der Öffentlichkeit zu übergeben.

\section{Eine Fehlerquelle bei der Bestimmung des Acetons nach der Jodoformmethode.}

Mitteilung aus dem Laboratorium von W. Vaubel zu Darmstadt.i

Von W. Vaterel und O. Scimetris.

(Eingeg. d. 18.'1. 1905.)

Die Bestimmung des Acetons nach der Jodoformmethode ist zuerst von $G$. $K \mathbf{r}$ ä $\mathrm{mer}^{\mathbf{1}}$ ) vor-

1) G. Krämer, Berl. Ber. 13, $1000(1880)$. geschlagen worden, und zwar zur Bestimmung des Acetongehaltes im Holzgeist. Hierbei kam das gebildete Jodoform als solches zur Wägung, und durfte der Gehalt an Aceton $1 \%$ nicht übersteigen. Diese Nethode erfuhr eine Abänderung durch J. Mess ing e r2). Nach dessen Methode verfährt man so, daß man die acetonhaltige Lösung mit 5-6\%:iger Kalilange versetzt, eine titrierte Jodlösung im t'berschuß zugibt und nach dem Ansäuern mit Schwefelsäure und zwei Minuten langem Stehenlassen unter Verwendung von Stärkekleister als Indikator mittcls Thiosulfat zurïcktitriert.

Die Messingersche Methode entspricht in dieser Fassung noch nicht allen Bedürfnissen. Sie hat dementsprechend vielfach Abänderungen erfahren. Vor einiger Zeit sind wir auf eine besonders ins Gewicht fallende Fehlerquelle aufmerksam geworden, über die wir nachstehend kurz berichten wollen. Die Beobachtung. um die es sich hier handelt, ist bereits ror kurzem ron $\mathrm{F} \ddot{\mathrm{r}} \mathrm{s}$ te $\mathrm{r}$ und $\left.\mathrm{G} \mathrm{yr}^{3}\right)$ beschrieben

2) J. Messinger, Berl. Ber. 21, 33 66(1888).

3) F. Foerster u. K. Gyr, Z. f. Elektrochem. 9, 1 (1903). 
worden. Sie war uns aber, als wir mit unseren Versuchen begannen, nicht bekannt und ist von uns also ganz unabhängig von der Veröffentlichung der oben erwähnten Forscher bei Untersuchungen nach der Messingerschen Methode gemacht worden.

Die Utberschrift der Arbeit von F örster und G y r lautet: ,Uber die Einwirkung ron Jod auf Alkalien". Der wesentliche Inhalt der Arbeit ist nach dem Referat im Chemischen Centralblatt folgender :

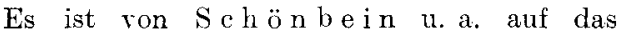
sicherste festgestellt worden, daß Jod mit Alkalien zunächst Hypojodit bildet. Es treten dabei nebeneinander die Gleichgewichte auf :

$$
\begin{gathered}
\mathrm{J}_{2}+\mathrm{OH}^{\prime}=\mathrm{HOJ}+\mathrm{J}^{\prime} . \\
\mathrm{HOJ}+\mathrm{OH}^{\prime}=\mathrm{JO}^{\prime}+\mathrm{H}_{2} \mathrm{O} .
\end{gathered}
$$

Daß die erste Reaktion unvollständig ist, und daß freies Jod neben freiem Alkali beständig ist, konnten Verf. sowohl direkt beweisen, als auch durch die Untersuchung, indem Zusatz von Jodkalium zu einer Lösung von unterjodiger Säure eine starke Jodausscheidung hervorruft. Unterjodige Säure ist wahrscheinlich eine noch schwächchere Säure als unterchlorige Säure. Die Menge des freien Jods neben einer bestimmten Menge Hydroxylionen ist im Sinne der Forderungen des Massenwirkungsgesetzes immer größer, je mehr Jodionen die Lösung enthält.

Setzt man Bicarbonat zu einer Lösung von Hypojodit, so wird dadurch die Menge des freien Jods vermindert, weil die Menge der Hydroxylionen vermindert wird. Es bleibt also immer eine gewisse Venge unterjodiger Säure zurück. Dies schließen die Verff. daraus, daß die Lösung weniger Thiosulfat verbraucht als arsenige Säure. Sie nehmen an, daB zwar das freie Jod Thio. sulfat zu Tetrathionat oxydiert, daß aber die unterjodige Säure das Thiosulfat direkt zu Sul. fat oxydiert. Arsenige Säure wird nicht durch freies Jod oxydiert, sondern nur durch unterjodige Säure. Gelöste Hypojodite gehen allmählich vollständig in Jodat ïber. Diese Reaktion erfolgt ganz analog der Reaktion des Chlorats nach der Gleichung

$$
\mathrm{KOJ}+2 \mathrm{HOJ}=\mathrm{KO}_{3} \mathrm{~J}+2 \mathrm{HJ} .
$$

Sie erfolgt mit sehr großer Geschwindigkeit und um so langsamer, je größer der Uberschuß an freiem Alkali ist. Temperaturerhöhung be. schleunigt die Reaktion. Auch die Vermehrung des freien Jodkaliums beschleunigt die Geschwindigkeit der Jodatbildung. Da durch die Jodatbildung die freie unterjodige Säure verbraucht wird, muß gleichzeitig anch das mit dieser im Gleichgewicht befindliche freie Jod verschwinden. Wenn neutrale Oxydationsmittel wie Sauerstoff, Ozon, Wasserstoffsuperoxyd, Perjodat usw. auf Jodkalium einwirken, entsteht neben dem freien Jod immer freies Alkali und durch deren Zwischenwirkung Hypojodit und Jodat. $\mathrm{Zu}$ beachten ist, dab durch die Reaktion des Hypojodits mit dem Wasserstoffsuperoxyd oder mit dem durch die Einwirkung von Ozon auf das
Atzkali entstandenen Kaliumsuperoxyd ein Teil des Hypojodits unter Sauerstoffentwicklung zerstört wird.

Wenn wir im vorstehenden die Resultate der von $\mathrm{F} \ddot{\mathrm{r}} \mathrm{st}$ e $\mathrm{r}$ und $\mathrm{G}$ y $\mathrm{r}$ ausgeführten Untersuchungen etwas ausführlicher wiedergegeben haben, so liegt der Grund darin, daß wir vorcrst von einer Erklärung der Tatsachen a.b. sehen und uns mit der Wirkung der beobachteten Erscheinungen auf die Acetonbestimmung begnïgen wollen.

Unsere bei der Acetonbestimmung gemachten Erfahrungen veranlaß3ten uns, die Einwirkung von Jod auf Alkali dureh einige Versuche klar zu legen. Dieselben ergaben folgendes:

Wenn man $20 \mathrm{ccm} 1 / 10^{-n}$. Satronlauge mit $20 \mathrm{cem} 1 / 10^{-n}$. Jodlösung zusammenbringt und mit Thiosulfat zurücktitriert, so werden direkt verbraucht $4,0 \mathrm{ccm} 1 / 10^{-n}$. Thiosulfatlösung und nach Zusatz von Essigsäure nochmals 13,2 , im ganzen also $17,2 \mathrm{ccm}$. Es fehlten also $2,8 \mathrm{~cm} 1 / 10^{-n}$. Thiosulfat, die noch verbraucht hätten werden müssen, wenn der Vorgang der Mischung von Jod auf Alkali unter Bildung von je einem Molekül Jodid und Hypojodit erfolgt sein würde. Zusatz von Schwefelsäure brachte keine Anderung.

Alsdann wurden $30 \mathrm{ccm} \mathrm{1/10-n.} \mathrm{Natronlauge}$ auf $20 \mathrm{ccm} 1 / 10-n$. Jodlösung verwendet. Es wurden vor dem Ansäuern $2,0 \mathrm{ccm} 1 / 10^{-n}$. Thiosulfat und nach dem Ansäuern noch $14,7 \mathrm{ccm} 1 / 10^{-n}$., im ganzen also $16,7 \mathrm{~cm} 1 / 10^{-n}$. Thiosulfat verbraucht. Die Menge des in der Natronlauge frei vorhandenen Jods war also gesunken, das Gesamtergebnis noch ungünstiger.

Verwendete man dagegen arsenige Säure zur Titration nach dem Ansäuern und Versetzen mit Bicarbonatlösung, so wurden richtige Resultate erhalten.

Umalsowenigstenséine Fehler. quelle bei der Messingersehen Methode der Acetonbestimmung z u vermeiden, ist es notwendig, mit arseniger Säure zurückzu titrieren und nicht mit Thiosulfat.

Die hier mitgeteilte Fehlerquelle scheint übrigens schon früher zur Abänderung der Versuchsanordnungen geführt zu haben. So empfiehlt M. Martz $z^{4}$ ) die Titerstellung des Thiosulfats auf eine bestimmte Menge Jod in Natronlauge gelöst und durch Säure wieder ausgeschieden. Hierdurch wird der Fehler teilweise vermieden werden können, jedoch nicht vollständig, da die oben mitgeteilten Versuche ergeben haben, daß die Größe des Fehlers mit den Verhältnissen von Jod zu Alkali wechselt

Mitteilungen über weitere Versuche über die titrimetrische Bestimmung des Acetons sollen folgen.

4) M. Martz, Rép. de Pharm. 1897,597; Pharm. Centralh. 38, 903; ‥ Chem. Centralbl. 1893 II, 238. 